\title{
Chaotic Tolerant Synchronization Analysis with Propagation Delay and Actuator Faults
}

\author{
Zhang Qunli \\ Department of Mathematics, Heze University, Heze, Shandong 274015, China \\ Correspondence should be addressed to Zhang Qunli; qunli-zhang@126.com \\ Received 20 June 2015; Accepted 24 August 2015 \\ Academic Editor: Laura Gardini
}

Copyright (c) 2015 Zhang Qunli. This is an open access article distributed under the Creative Commons Attribution License, which permits unrestricted use, distribution, and reproduction in any medium, provided the original work is properly cited.

The criteria for tolerant synchronization with a constant propagation delay and actuator faults are presented by using matrix analysis techniques. A new algorithm, which constructs the extended error systems in order to make the conservation of the stability lower, is proposed. Based on proper Lyapunov-Krasovskii functional, the novel delay-dependent fault tolerant synchronization analyses are derived. Finally, numerical examples show the effectiveness of the proposed method.

\section{Introduction}

The realization of OGY (Ott-Grebogi-Yorker) chaos-control method [1] and PC (Pecora and Carroll) synchronization method [2] has been attracting researchers' attention since the 1990s. Chaos synchronization [3-13] is of great practical significance and has aroused great interest in recent years.

They all focused on the design of synchronization under normal operating conditions in the above works described. But, in practical chaotic secure communication systems, sensors, actuators, and inner components may inevitably fail, which can lead to sharp performance decline of chaotic secure. For the reason, fault tolerant synchronization and control [14-20] of chaotic systems have been the hot topic of intensive researches recently.

More recent works studied the fault tolerant synchronization and control, but they were limited to construct Lyapunov-Krasovskii functional

$$
V(t)=V_{1}(e(t))+V_{2}(\tilde{f}(t))
$$

which separates $e(t)$ and $\widetilde{f}(t)$, where $e(t)$ is the synchronization error and $\widetilde{f}(t)$ is the error of fault function. Motivating the limitation and the extended transformation [21, 22], the effective new method, which does the extended transformation for the error system, to consider Lyapunov-Krasovskii functional

$$
V(t)=V\left(\left(e^{T}(t), \tilde{f}^{T}(t)\right)^{T}\right),
$$

which do not separate $e(t)$ and $\widetilde{f}(t)$, for the chaotic fault tolerant synchronization with a constant propagation delay and actuator faults, makes the conservation descent. Finally, numerical examples are given to verify the above method.

Notations. In this paper, $R, R^{n}$, and $R^{n \times m}$ denote, respectively, the real number, the real $n$-vectors, and the real $n \times m$ matrices. The superscript " $T$ " stands for the transpose of a matrix. The symbol $X>Y(X \geq Y)$, where $X$ and $Y$ are symmetric matrices, means that $X-Y$ is positive definite (positive semidefinite). $I$ is the identity matrix of appropriate dimensions. "*" denotes the matrix entries implied by symmetry.

\section{Preliminaries and Systems Description}

Consider a chaotic master system with the actuator faults item $f(t)$ in the following form:

$$
\begin{aligned}
& \dot{x}(t)=A x(t)+g(x(t))+h(x(t-\theta))+E f(t), \\
& y(t)=C x(t)+D f(t),
\end{aligned}
$$


where $x(t) \in R^{n}$ is the measurable state vector. $y(t) \in R^{p}$ is the output vector, and $A, C, D$, and $E$ are proper dimension constant matrices. $g(x(t)), h(x(t-\theta))$ are known continuous nonlinear functions. $\theta>0$ is the delay.

Assumption 1. There exist the matrices $U_{1}, U_{2}, M_{1}, M_{2}$, $W_{1}, W_{2}, V_{1}, V_{2} \in R^{n \times n}$, and the nonlinear functions $g(\cdot)$, and $h(\cdot)$ satisfy

$$
\begin{aligned}
& \left(U_{1}(g(x)-g(y))-U_{2}(x-y)\right)^{T} \\
& \quad \cdot\left(M_{1}(g(x)-g(y))-M_{2}(x-y)\right) \leq 0, \\
& \left(W_{1}(h(x)-h(y))-W_{2}(x-y)\right)^{T} \\
& \quad \cdot\left(V_{1}(h(x)-h(y))-V_{2}(x-y)\right) \leq 0
\end{aligned}
$$

for all $x, y \in R^{n}$.

The slave system linked with the chaotic master system (3) is described by

$$
\begin{aligned}
\dot{\hat{x}}(t)= & A \widehat{x}(t)+g(\widehat{x}(t))+h(\widehat{x}(t-\theta))+E \widehat{f} \\
& -L(\widehat{y}(t-\tau)-y(t-\tau)), \\
\hat{y}(t)= & C \widehat{x}(t)+D \widehat{f}(t),
\end{aligned}
$$

where $\widehat{x}(t) \in R^{n}$ is the measurable state vector and $\tau>0$ is a constant propagation delay.

Let $e(t)=\widehat{x}(t)-x(t), \widetilde{f}(t)=\widehat{f}(t)-f(t)$, and $\varphi(t)=$ $\hat{y}(t)-y(t)=C e(t)+D \tilde{f}(t)$, and then

$$
\begin{aligned}
\dot{e}(t)= & A e(t)+g(\widehat{x}(t))-g(x(t))+h(\widehat{x}(t-\theta)) \\
& -h(x(t-\theta))+E \tilde{f}(t)-L C e(t-\tau) \\
& -L D \tilde{f}(t-\tau), \\
\varphi(t)= & C e(t)+D \tilde{f}(t) .
\end{aligned}
$$

Lemma 2 (see $[23,24])$. For any constant matrix $W \in R^{n \times n}$, $W>0$, scalar $0<h(t)<h$, and vector function $w(t)$ : $[0, h] \rightarrow R^{n}$ such that the integrations concerned are well defined, and then

$$
\begin{gathered}
\left(\int_{0}^{h(t)} w(s) d s\right)^{T} W\left(\int_{0}^{h(t)} w(s) d s\right) \\
\leq h(t) \int_{0}^{h(t)} w^{T}(s) W w(s) d s .
\end{gathered}
$$

Lemma 3 (see [25]). Let $Q>0, H, F(t)$, and $E$ be real matrices of appropriate dimensions, with $F(t)$ satisfying $F^{T}(t) F(t) \leq$ I. Then, the following inequalities are equivalent:

(1) $Q+H F(t) E+E^{T} F^{T}(t) H^{T}<0$;

(2) there exists a scalar $\varepsilon>0$ such that $Q+\varepsilon^{-1} H H^{T}+$ $\varepsilon E^{T} E<0$.

Lemma 4 (see [26-28]). Let $A, L, E$, and $F(t)$ be real matrices of appropriate dimensions, with $F(t)$ satisfying $F^{T}(t) F(t) \leq I$. Then, one has the following:
(1) for any scalar $\varepsilon>0$,

$$
L F E+E^{T} F^{T} L^{T}<\varepsilon^{-1} L L^{T}+\varepsilon E^{T} E
$$

(2) for any matrix $P>0$ and scalar $\varepsilon>0$, such that $\varepsilon I-$ $E F E^{T}>0$,

$$
\begin{aligned}
(A & +L F E)^{T} P(A+L F E) \\
& \leq A^{T} P A+A^{T} P E\left(\varepsilon I-E^{T} P E\right)^{-1} E^{T} P A+\varepsilon L^{T} L .
\end{aligned}
$$

\section{Fault Tolerant Synchronization Analysis}

3.1. Fault Tolerant Synchronization Analysis When $f(t)$ and $\widehat{f}(t)$ Are Derivable on $t$. Let $\dot{\tilde{f}}(t)=-G \varepsilon(t)=-G C e(t)-$ $G D \widetilde{f}(t)$, and we have

$$
\begin{aligned}
\dot{e}(t)= & A e(t)+g(\widehat{x}(t))-g(x(t))+h(\widehat{x}(t-\theta)) \\
& -h(x(t-\theta))+E \tilde{f}(t)-L C e(t)+L C e(t) \\
& -L C e(t-\tau)-L D \tilde{f}(t)+L D \tilde{f}(t) \\
& -L D \tilde{f}(t-\tau) \\
= & (A-L C) e(t)+g(\widehat{x}(t))-g(x(t)) \\
& +h(\widehat{x}(t-\theta))-h(x(t-\theta))+(E-L D) \tilde{f}(t) \\
& +L C \int_{t-\tau}^{t} \dot{e}(s) d s+L D \int_{t-\tau}^{t} \dot{\tilde{f}}(s) d s .
\end{aligned}
$$

Suppose $\eta(t)=\left(\begin{array}{l}e(t) \\ \tilde{f}(t)\end{array}\right)$, and then

$$
\begin{aligned}
\dot{e}(t)= & (A-L C, E-L D)\left(\begin{array}{c}
e(t) \\
\tilde{f}(t)
\end{array}\right)+g(\widehat{x}(t)) \\
& -g(x(t))+h(\widehat{x}(t-\theta))-h(x(t-\theta)) \\
& +(L C, L D)\left(\begin{array}{c}
\int_{t-\tau}^{t} \dot{e}(s) d s \\
\int_{t-\tau}^{t} \dot{\tilde{f}}(s) d s
\end{array}\right) \\
= & (A-L C, E-L D) \eta(t)+g(\widehat{x}(t)) \\
& -g(x(t))+(L C, L D) \int_{t-\tau}^{t} \dot{\eta}(s) d s, \\
\dot{\tilde{f}}(t)= & (-G C,-G D)\left(\begin{array}{c}
e(t) \\
\tilde{f}(t)
\end{array}\right)=(-G C,-G D) \eta(t),
\end{aligned}
$$




$$
\begin{aligned}
\dot{\eta}(t)= & \left(\begin{array}{cc}
A-L C & E-L D \\
-G C & -G D
\end{array}\right) \eta(t) \\
& +\left(\begin{array}{c}
g(\widehat{x}(t))-g(x(t)) \\
0
\end{array}\right) \\
& +\left(\begin{array}{c}
h(\widehat{x}(t-\theta))-h(x(t-\theta)) \\
0
\end{array}\right) \\
& +\left(\begin{array}{cc}
L C & L D \\
0 & 0
\end{array}\right) \int_{t-\tau}^{t} \dot{\eta}(s) d s, \\
\dot{\eta}(t)= & B \eta(t)+\left(\begin{array}{c}
p(t) \\
0
\end{array}\right)+\left(\begin{array}{c}
q(t) \\
0
\end{array}\right)+R \int_{t-\tau}^{t} \dot{\eta}(s) d s
\end{aligned}
$$

where

$$
\begin{aligned}
B & =\left(\begin{array}{cc}
A-L C & E-L D \\
-G C & -G D
\end{array}\right), \\
p(t) & =g(\widehat{x}(t))-g(x(t)), \\
q(t) & =h(\hat{x}(t-\theta))-h(x(t-\theta)), \\
R & =\left(\begin{array}{cc}
L C & L D \\
0 & 0
\end{array}\right) .
\end{aligned}
$$

From the assumption, we have

$$
\begin{aligned}
(g(x) & -g(y))^{T} U_{1}^{T} M_{1}(g(x)-g(y)) \\
& -(g(x)-g(y))^{T} U_{1}^{T} M_{2}(x-y) \\
& -(x-y)^{T} U_{2}^{T} M_{1}(g(x)-g(y)) \\
& +(x-y)^{T} U_{2}^{T} M_{2}(x-y) \leq 0 .
\end{aligned}
$$

So, we can get from assumption that

$$
\begin{aligned}
& (p(t))^{T} U_{1}^{T} M_{1} p(t)-(p(t))^{T} U_{1}^{T} M_{2} e(t) \\
& -(e(t))^{T} U_{2}^{T} M_{1} p(t)+(e(t))^{T} U_{2}^{T} M_{2} e(t) \leq 0, \\
& \left(\begin{array}{lll}
e^{T}(t) & \tilde{f}^{T}(t)
\end{array}\right)\left(\begin{array}{cc}
-U_{2}^{T} M_{2} & 0 \\
0 & 0
\end{array}\right)\left(\begin{array}{c}
e(t) \\
\tilde{f}(t)
\end{array}\right) \\
& +\left(\begin{array}{ll}
e^{T}(t) & \widetilde{f}^{T}(t)
\end{array}\right)\left(\begin{array}{cc}
U_{2}^{T} M_{1} & R_{1} \\
0 & R_{2}
\end{array}\right)\left(\begin{array}{c}
p(t) \\
0
\end{array}\right) \\
& +\left(\begin{array}{ll}
p^{T}(t) & 0
\end{array}\right)\left(\begin{array}{cc}
U_{1}^{T} M_{2} & 0 \\
R_{3} & R_{4}
\end{array}\right)\left(\begin{array}{c}
e(t) \\
\tilde{f}(t)
\end{array}\right) \\
& +\left(\begin{array}{ll}
p^{T}(t) & 0
\end{array}\right)\left(\begin{array}{cc}
-U_{1}^{T} M_{1} & R_{5} \\
R_{6} & R_{7}
\end{array}\right)\left(\begin{array}{c}
p(t) \\
0
\end{array}\right) \geq 0,
\end{aligned}
$$

in which the proper dimension matrices $R_{i} \in R^{n \times n}, i=$ $1,2, \ldots, 7$, are arbitrary.
That is,

$$
\begin{aligned}
& \eta^{T}(t)\left(\begin{array}{cc}
-U_{2}^{T} M_{2} & 0 \\
0 & 0
\end{array}\right) \eta(t) \\
& +\eta^{T}(t)\left(\begin{array}{cc}
U_{2}^{T} M_{1} & R_{1} \\
0 & R_{2}
\end{array}\right)\left(\begin{array}{c}
p(t) \\
0
\end{array}\right) \\
& +\left(\begin{array}{c}
p(t) \\
0
\end{array}\right)^{T}\left(\begin{array}{cc}
U_{1}^{T} M_{2} & 0 \\
R_{3} & R_{4}
\end{array}\right) \eta(t) \\
& +\left(\begin{array}{c}
p(t) \\
0
\end{array}\right)^{T}\left(\begin{array}{cc}
-U_{1}^{T} M_{1} & R_{5} \\
R_{6} & R_{7}
\end{array}\right)\left(\begin{array}{c}
p(t) \\
0
\end{array}\right) \geq 0 \\
& \eta^{T}(t)\left(\begin{array}{c}
\left.\frac{1}{2}\left(S_{1}+S_{1}^{T}\right)\right) \eta(t)
\end{array}\right.
\end{aligned}
$$

$$
\begin{aligned}
& +\eta^{T}(t)\left(\frac{1}{2}\left(S_{2}+S_{3}^{T}\right)\right)\left(\begin{array}{c}
p(t) \\
0
\end{array}\right) \\
& +\left(\begin{array}{c}
p(t) \\
0
\end{array}\right)^{T}\left(\frac{1}{2}\left(S_{3}+S_{2}^{T}\right)\right) \eta(t) \\
& +\left(\begin{array}{c}
p(t) \\
0
\end{array}\right)^{T}\left(\frac{1}{2}\left(S_{4}+S_{4}^{T}\right)\right)\left(\begin{array}{c}
p(t) \\
0
\end{array}\right) \geq 0,
\end{aligned}
$$

where

$$
\begin{aligned}
& S_{1}=\left(\begin{array}{cc}
-\varepsilon U_{2}^{T} M_{2} & 0 \\
0 & 0
\end{array}\right), \\
& S_{2}=\left(\begin{array}{cc}
\varepsilon U_{2}^{T} M_{1} & \varepsilon R_{1} \\
0 & \varepsilon R_{2}
\end{array}\right), \\
& S_{3}=\left(\begin{array}{cc}
\varepsilon U_{1}^{T} M_{2} & 0 \\
\varepsilon R_{3} & \varepsilon R_{4}
\end{array}\right), \\
& S_{4}=\left(\begin{array}{cc}
-\varepsilon U_{1}^{T} M_{1} & \varepsilon R_{5} \\
\varepsilon R_{6} & \varepsilon R_{7}
\end{array}\right), \\
& \quad \varepsilon>0
\end{aligned}
$$

is any constant. Consider

$$
\begin{aligned}
& \eta^{T}(t)\left(S_{1}+S_{1}^{T}\right) \eta(t)+\eta^{T}(t)\left(S_{2}+S_{3}^{T}\right)\left(\begin{array}{c}
p(t) \\
0
\end{array}\right) \\
& +\left(\begin{array}{c}
p(t) \\
0
\end{array}\right){ }^{T}\left(S_{3}+S_{2}^{T}\right) \eta(t) \\
& \quad+\left(\begin{array}{c}
p(t) \\
0
\end{array}\right)^{T}\left(S_{4}+S_{4}^{T}\right)\left(\begin{array}{c}
p(t) \\
0
\end{array}\right) \geq 0,
\end{aligned}
$$

$\xi^{T}(t) \Lambda_{1} \xi(t) \geq 0$ 
where

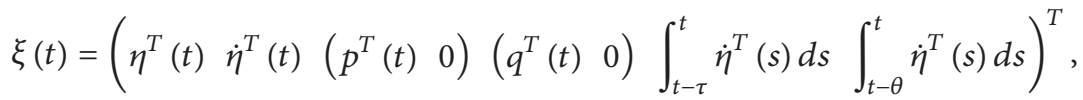

$$
\begin{aligned}
& \Lambda_{1}=\left(\begin{array}{cccccc}
S_{1}+S_{1}^{T} & 0 & 0 & S_{2}+S_{3}^{T} & 0 & 0 \\
* & 0 & 0 & 0 & 0 & 0 \\
* & * & S_{4}+S_{4}^{T} & 0 & 0 & 0 \\
* & * & * & 0 & 0 & 0 \\
* & * & * & * & 0 & 0 \\
* & * & * & * & * & 0
\end{array}\right) .
\end{aligned}
$$

Imitating the above inference, we obtain from inequality (5) the following:

$$
\xi^{T}(t) \Lambda_{2} \xi(t) \geq 0
$$

where

$$
\Lambda_{2}=\delta\left(\begin{array}{ccccccc}
Y_{1}^{T} \Delta Y_{1} & 0 & 0 & Y_{1}^{T} \Delta Y_{3} & 0 & Y_{1}^{T} \Delta Y_{2} \\
* & 0 & 0 & 0 & 0 & 0 \\
* & * & 0 & 0 & 0 & 0 \\
* & * & * & Y_{3}^{T} \Delta Y_{3} & 0 & Y_{3}^{T} \Delta Y_{2} \\
* & * & * & * & 0 & 0 & \\
* & * & * & * & * & Y_{2}^{T} \Delta Y_{2}
\end{array}\right),
$$

We choose Lyapunov-Krasovskii functional

$$
\begin{aligned}
V(t)= & \eta^{T}(t) P \eta(t)+\int_{-\tau}^{0} \int_{t+r}^{t} \dot{\eta}^{T}(s) Q \dot{\eta}(s) d s d r \\
& +\int_{-\theta}^{0} \int_{t+r}^{t} \dot{\eta}^{T}(s) \Theta \dot{\eta}(s) d s d r .
\end{aligned}
$$

Differentiating $V(t)$ with respect to $t$, the following result is yielded:

$$
\begin{aligned}
\dot{V}(t)= & 2 \eta^{T}(t) P \dot{\eta}(t)+\tau \dot{\eta}^{T}(t) Q \dot{\eta}(t) \\
& -\int_{t-\tau}^{t} \dot{\eta}^{T}(s) Q \dot{\eta}(s) d s \\
= & 2 \eta^{T}(t) P B \eta(t)+2 \eta^{T}(t) P\left(\begin{array}{c}
p(t) \\
0
\end{array}\right) \\
& +2 \eta^{T}(t) P R \int_{t-\tau}^{t} \dot{\eta}(s) d s+\tau \dot{\eta}^{T}(t) Q \dot{\eta}(t) \\
& -\int_{t-\tau}^{t} \dot{\eta}^{T}(s) Q \dot{\eta}(s) d s+\theta \dot{\eta}^{T}(t) \Theta \dot{\eta}(t) \\
& -\int_{t-\theta}^{t} \dot{\eta}^{T}(s) \Theta \dot{\eta}(s) d s .
\end{aligned}
$$

From Lemma 2, we have

$$
\begin{aligned}
\dot{V}(t) \leq & \eta^{T}(t)\left(P B+B^{T} P\right) \eta(t)+2 \eta^{T}(t) P\left(\begin{array}{c}
p(t) \\
0
\end{array}\right) \\
& +2 \eta^{T}(t) P\left(\begin{array}{c}
q(t) \\
0
\end{array}\right)+2 \eta^{T}(t) P R \int_{t-\tau}^{t} \dot{\eta}(s) d s \\
& +\tau \dot{\eta}^{T}(t) Q \dot{\eta}(t) \\
& -\frac{1}{\tau}\left(\int_{t-\tau}^{t} \dot{\eta}(s) d s\right)^{T} Q\left(\int_{t-\tau}^{t} \dot{\eta}(s) d s\right) \\
& +\theta \dot{\eta}^{T}(t) \Theta \dot{\eta}(t) \\
& -\frac{1}{\theta}\left(\int_{t-\tau}^{t} \dot{\eta}(s) d s\right)^{T} \Theta\left(\int_{t-\tau}^{t} \dot{\eta}(s) d s\right) \\
= & \xi^{T}(t) \Pi \xi(t) \geq 0,
\end{aligned}
$$


where

$$
\Pi=\left(\begin{array}{cccccc}
P B+B^{T} P & 0 & P & P & P R & 0 \\
* & \tau Q & 0 & 0 & 0 & 0 \\
* & * & 0 & 0 & 0 & 0 \\
* & * & * & 0 & 0 & 0 \\
* & * & * & * & -\frac{1}{\tau} Q & 0 \\
* & * & * & * & * & -\frac{1}{\theta} \Theta
\end{array}\right) .
$$

From model (12), we have

$$
\begin{aligned}
0= & 2\left(Q_{1} \eta(t)+Q_{2} \dot{\eta}(t)\right)^{T} \\
& \cdot\left(-\dot{\eta}(t)+B \eta(t)+\left(\begin{array}{c}
p(t) \\
0
\end{array}\right)+R \int_{t-\tau}^{t} \dot{\eta}(s) d s\right) \\
= & \xi^{T}(t) \Xi \xi(t),
\end{aligned}
$$

where

$$
\Xi\left(\begin{array}{cccccc}
Q_{1}^{T} B+B^{T} Q_{1} & -Q_{1}^{T}+B^{T} Q_{2}^{T} & Q_{1}^{T} & Q_{1}^{T} & Q_{1}^{T} R & 0 \\
* & -Q_{2}^{T}-Q_{2} & Q_{2}^{T} & Q_{2}^{T} & Q_{2}^{T} R & 0 \\
* & * & 0 & 0 & 0 & 0 \\
* & * & * & 0 & 0 & 0 \\
* & * & * & * & 0 & 0 \\
* & * & * & * & * & 0
\end{array}\right) .
$$

From formulas (18), (20), (24), and (26), we get

$$
\begin{aligned}
\dot{V}(t) & \leq \xi^{T}(t)\left(\Lambda_{1}+\Lambda_{2}+\Pi+\Xi\right) \xi(t) \\
& =\xi^{T}(t) \Omega \xi(t),
\end{aligned}
$$

where $\Omega=\Lambda+\Pi+\Xi$.

Based on the above derivation, we have the following result.

Theorem 5. The fault tolerant synchronization (3) and (6) is achieved if there exist constants $\tau>0, \theta>0, \delta>0$, and $\varepsilon>0$, the positive definite matrices $P=P^{T}>0, Q=Q^{T}>0$, and $\Theta>0$, and the matrices $Q_{j}, M_{j}, U_{j}, j=1,2, L, C, D, R_{i}$, $i=1,2, \ldots, 8$, such that the matrix $\Omega<0$.

Remark 6. After the extended transformation $e(t) \rightarrow \eta(t)$, system (7) is turned into system (12). Based on the Lyapunov functional in [15-21], we take Lyapunov functional

$$
\begin{aligned}
V(t)= & \eta^{T}(t) P \eta(t)+\int_{-\tau}^{0} \int_{t+r}^{t} \dot{\eta}^{T}(s) Q \dot{\eta}(s) d s d r \\
& +\int_{-\theta}^{0} \int_{t+r}^{t} \dot{\eta}^{T}(s) \Theta \dot{\eta}(s) d s d r,
\end{aligned}
$$

and we get matrix $\Omega$. The conservation of stability of error system (7) can be decreased by choosing the matrices $R_{i}, i=$ $1,2, \ldots, 8$, and the constant $\varepsilon>0$.
Corollary 7. When $D=0$ in system (3), result similar to Theorem 5 can be obtained.

When $h(x(t-\theta))=0$, inequality (18) is transformed to

$$
\sigma^{T}(t) \Lambda \sigma(t) \geq 0
$$

where

$\sigma(t)$

$$
\begin{aligned}
& =\left(\eta^{T}(t) \dot{\eta}^{T}(t)\left(\begin{array}{c}
p(t) \\
0
\end{array}\right)^{T}\left(\int_{t-\tau}^{t} \dot{\eta}(s) d s\right)^{T}\right)^{T}, \\
& \Lambda=\left(\begin{array}{cccc}
S_{1}+S_{1}^{T} & 0 & S_{2}+S_{3}^{T} & 0 \\
* & 0 & 0 & 0 \\
* & * & S_{4}+S_{4}^{T} & 0 \\
* & * & * & 0
\end{array}\right), \\
& S_{1}=\left(\begin{array}{cc}
-\varepsilon U_{2}^{T} M_{2} & 0 \\
0 & 0
\end{array}\right) \\
& S_{2}=\left(\begin{array}{cc}
\varepsilon U_{2}^{T} M_{1} & \varepsilon R_{1} \\
0 & \varepsilon R_{2}
\end{array}\right) \text {, } \\
& S_{3}=\left(\begin{array}{cc}
\varepsilon U_{1}^{T} M_{2} & 0 \\
\varepsilon R_{3} & \varepsilon R_{4}
\end{array}\right) \text {, } \\
& S_{4}=\left(\begin{array}{cc}
-\varepsilon U_{1}^{T} M_{1} & \varepsilon R_{5} \\
\varepsilon R_{6} & \varepsilon R_{7}
\end{array}\right) \text {, } \\
& \varepsilon>0
\end{aligned}
$$

is any constant. The proper dimension matrices $R_{i} \in R^{n \times n}$, $i=1,2, \ldots, 7$, are arbitrary.

We choose Lyapunov functional

$$
V(t)=\eta^{T}(t) P \eta(t)+\int_{-\tau}^{0} \int_{t+\theta}^{t} \dot{\eta}^{T}(s) Q \dot{\eta}(s) d s d \theta
$$

and differentiating $V(t)$ with respect to $t$ and using Lemma 2 yield

$$
\dot{V}(t) \leq \sigma^{T}(t) \Pi \sigma(t)
$$

where

$$
\Pi=\left(\begin{array}{cccc}
P B+B^{T} P & 0 & P & P R \\
* & \tau Q & 0 & 0 \\
* & * & 0 & 0 \\
* & * & * & -\frac{1}{\tau} Q
\end{array}\right) .
$$


From model (12), we have

$$
\begin{aligned}
0= & 2\left(Q_{1} \eta(t)+Q_{2} \dot{\eta}(t)\right)^{T} \\
& \cdot\left(-\dot{\eta}(t)+B \eta(t)+\left(\begin{array}{c}
p(t) \\
0
\end{array}\right)+R \int_{t-\tau}^{t} \dot{\eta}(s) d s\right) \\
& =\sigma^{T}(t) \Xi \sigma(t),
\end{aligned}
$$

where

$$
\Xi=\left(\begin{array}{cccc}
Q_{1}^{T} B+B^{T} Q_{1} & -Q_{1}^{T}+B^{T} Q_{2}^{T} & Q_{1}^{T} & Q_{1}^{T} R \\
* & -Q_{2}^{T}-Q_{2} & Q_{2}^{T} & Q_{2}^{T} R \\
* & * & 0 & 0 \\
* & * & * & 0
\end{array}\right)
$$

Based on the above derivation, we have the following result.

Corollary 8. The fault tolerant synchronization (3) and (5) is achieved if there exist constants $\tau, \varepsilon>0$, the positive definite matrices $P=P^{T}>0, Q=Q^{T}>0$, and the matrices $Q_{j}, M_{j}, U_{j}, j=1,2, Q_{j}, M_{j}, U_{j}, j=1,2, L, C, D, R_{i}, i=$ $1,2, \ldots, 7$, such that matrix $\Omega<0$.

3.2. Fault Tolerant Synchronization Analysis When $f(t)$ and $\widehat{f}(t)$ Are Derivable on $x(t)$. Let $d f(t) / d x(t)=\hbar(x(t))=$ $H \cdot F(x(t)) \cdot N$ and $d \widehat{f}(t) / d x(t)=\hbar(\widehat{x}(t))=H \cdot F(\widehat{x}(t))$. $N, H, N$ be proper dimension matrices, with satisfying $F^{T}(x(t)) F(x(t)) \leq I$, where $H, N$ are proper dimension matrices and $I$ is identity matrix. Then,

$$
\begin{aligned}
\dot{e}(t)= & A e(t)+g(\hat{x}(t))-g(x(t))+h(\widehat{x}(t-\theta)) \\
& -h(x(t-\theta))+E \tilde{f}(t)-L C e(t)+L C e(t) \\
& -L C e(t-\tau)-L D \tilde{f}(t)+L D \tilde{f}(t) \\
& -L D \tilde{f}(t-\tau) \\
= & (A-L C) e(t)+g(\widehat{x}(t))-g(x(t)) \\
& +(E-L D) \tilde{f}(t)+L C \int_{t-\tau}^{t} \dot{e}(s) d s \\
& +L D \int_{t-\tau}^{t} \dot{\tilde{f}}(s) d s .
\end{aligned}
$$

Suppose $\eta(t)=\left(\begin{array}{c}e(t) \\ \tilde{f}(t)\end{array}\right)$; we have

$$
\begin{aligned}
\dot{e}(t)= & (A-L C, E-L D)\left(\begin{array}{c}
e(t) \\
\tilde{f}(t)
\end{array}\right)+g(\hat{x}(t)) \\
& -g(x(t))+h(\hat{x}(t-\theta))-h(x(t-\theta))
\end{aligned}
$$

$$
\begin{aligned}
& +(L C, L D)\left(\begin{array}{c}
\int_{t-\tau}^{t} \dot{e}(s) d s \\
\int_{t-\tau}^{t} \dot{\tilde{f}}(s) d s
\end{array}\right) \\
& =(A-L C, E-L D) \eta(t)+g(\widehat{x}(t))-g(x(t)) \\
& +h(\widehat{x}(t-\theta))-h(x(t-\theta)) \\
& +(L C, L D) \int_{t-\tau}^{t} \dot{\eta}(s) d s .
\end{aligned}
$$

According to $d f(t) / d x(t)=\hbar(x(t))=H \cdot F(x(t)) \cdot N$, $d \widehat{f}(t) / d x(t)=\hbar(\widehat{x}(t))=H \cdot F(\widehat{x}(t)) \cdot N$, we get

$$
\begin{aligned}
& \tilde{f}(t)=\int_{0}^{1} \hbar((1-\lambda) x(t)+\lambda \widehat{x}(t)) e(t) d \lambda \\
& =\int_{0}^{1} H \cdot F((1-\lambda) x(t)+\lambda \widehat{x}(t)) \cdot N \cdot e(t) d \lambda, \\
& -\delta \tilde{f}(t) \\
& \quad+\delta \int_{0}^{1} H \cdot F((1-\lambda) x(t)+\lambda \widehat{x}(t)) \cdot N \cdot e(t) d \lambda \\
& \quad=0 \\
& \left(\delta \int_{0}^{1} H \cdot F((1-\lambda) x(t)+\lambda \widehat{x}(t)) \cdot N d \lambda,-\delta I\right) \eta(t) \\
& \quad=0
\end{aligned}
$$

where $\delta>0$. Consider

$$
\begin{aligned}
& \left(\begin{array}{ll}
I & 0 \\
0 & 0
\end{array}\right) \dot{\eta}(t) \\
& =\left(\begin{array}{cc}
A-L C & E-L D \\
\delta \int_{0}^{1} H \cdot F((1-\lambda) x(t)+\lambda \widehat{x}(t)) \cdot N d \lambda & -\delta I
\end{array}\right) \eta(t) \\
& +\left(\begin{array}{cc}
g(\widehat{x}(t))-g(x(t)) \\
0
\end{array}\right)+\left(\begin{array}{cc}
L C & L D \\
0 & 0
\end{array}\right) \int_{t-\tau}^{t} \dot{\eta}(s) d s, \\
& E_{1} \dot{\eta}(t)=B \eta(t)+\left(\begin{array}{c}
p(t) \\
0
\end{array}\right)+\left(\begin{array}{c}
q(t) \\
0
\end{array}\right)+R \int_{t-\tau}^{t} \dot{\eta}(s) d s,
\end{aligned}
$$

where

$$
\begin{aligned}
& E_{1}=\left(\begin{array}{ll}
I & 0 \\
0 & 0
\end{array}\right), \\
& B
\end{aligned}
$$

$$
\begin{aligned}
& =\left(\begin{array}{cc}
A-L C & E-L D \\
\delta \int_{0}^{1} H \cdot F((1-\lambda) x(t)+\lambda \hat{x}(t)) \cdot N d \lambda & -\delta I
\end{array}\right), \\
& p(t)=g(\widehat{x}(t))-g(x(t)), \\
& R=\left(\begin{array}{cc}
L C & L D \\
0 & 0
\end{array}\right) .
\end{aligned}
$$


From the assumption, we have

$$
\varsigma^{T}(t) \Lambda_{1} \varsigma(t) \geq 0
$$

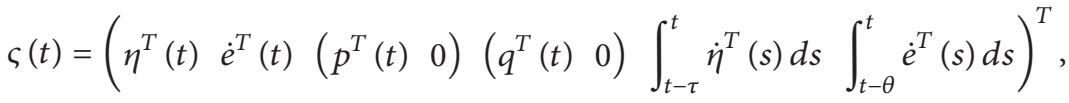

$$
\begin{aligned}
& \Lambda_{1}=\left(\begin{array}{cccccc}
S_{1}+S_{1}^{T} & 0 & 0 & S_{2}+S_{3}^{T} & 0 & 0 \\
* & 0 & 0 & 0 & 0 & 0 \\
* & * & S_{4}+S_{4}^{T} & 0 & 0 & 0 \\
* & * & * & 0 & 0 & 0 \\
* & * & * & * & 0 & 0 \\
* & * & * & * & * & 0
\end{array}\right),
\end{aligned}
$$

is any constant. The proper dimension matrices $R_{i} \in R^{n \times n}$, $i=1,2, \ldots, 7$, are arbitrary

$$
\varsigma^{T}(t) \Lambda_{2} \varsigma(t) \geq 0
$$

where

$\Lambda_{2}$

$$
=\delta\left(\begin{array}{cccccc}
Y_{1}^{T} \Delta Y_{1} & 0 & 0 & Y_{1}^{T} \Delta Y_{3} & 0 & Y_{1}^{T} \Delta Y_{2} \\
* & 0 & 0 & 0 & 0 & 0 \\
* & * & 0 & 0 & 0 & 0 \\
* & * & * & Y_{3}^{T} \Delta Y_{3} & 0 & Y_{3}^{T} \Delta Y_{2} \\
* & * & * & * & 0 & 0 \\
* & * & * & * & * & -W_{2}^{T} V_{2}-V_{2}^{T} W_{2}
\end{array}\right),
$$

where

$$
\begin{aligned}
& S_{1}=\left(\begin{array}{cc}
-\varepsilon U_{2}^{T} M_{2} & 0 \\
0 & 0
\end{array}\right), \\
& S_{2}=\left(\begin{array}{cc}
\varepsilon U_{2}^{T} M_{1} & \varepsilon R_{1} \\
0 & \varepsilon R_{2}
\end{array}\right), \\
& S_{3}=\left(\begin{array}{cc}
\varepsilon U_{1}^{T} M_{2} & 0 \\
\varepsilon R_{3} & \varepsilon R_{4}
\end{array}\right), \\
& S_{4}=\left(\begin{array}{cc}
-\varepsilon U_{1}^{T} M_{1} & \varepsilon R_{5} \\
\varepsilon R_{6} & \varepsilon R_{7}
\end{array}\right), \\
& \varepsilon>0
\end{aligned}
$$

$$
\begin{gathered}
Y_{1}=\left(\begin{array}{ll}
I & 0 \\
0 & 0
\end{array}\right), \\
Y_{2}=\left(\begin{array}{ll}
-I & 0 \\
0 & 0
\end{array}\right), \\
Y_{3}=\left(\begin{array}{ll}
0 & 0 \\
I & R_{8}
\end{array}\right),
\end{gathered}
$$

$$
\Delta=\left(\begin{array}{cc}
-W_{2}^{T} V_{2}-V_{2}^{T} W_{2} & W_{2}^{T} V_{1}+V_{2}^{T} W_{1} \\
* & -W_{1}^{T} V_{1}-V_{1}^{T} W_{1}
\end{array}\right)
$$

We choose Lyapunov function

$$
\begin{aligned}
V(t)= & e^{T}(t) P_{1} e(t)+\int_{-\tau}^{0} \int_{t+r}^{t} \dot{e}^{T}(s) Q \dot{e}(s) d s d r \\
& +\int_{-\theta}^{0} \int_{t+r}^{t} \dot{e}^{T}(s) \Theta \dot{e}(s) d s d r
\end{aligned}
$$




$$
\begin{aligned}
= & \eta^{T}(t) P E_{1} \eta(t)+\int_{-\tau}^{0} \int_{t+\theta}^{t} \dot{e}^{T}(s) Q \dot{e}(s) d s d \theta \\
& +\int_{-\theta}^{0} \int_{t+r}^{t} \dot{e}^{T}(s) \Theta \dot{e}(s) d s d r
\end{aligned}
$$

where $P=\left(\begin{array}{cc}P_{1} & P_{2} \\ 0 & P_{3}\end{array}\right)$, and it is easy to know that $P E_{1}=E_{1}^{T} P^{T}$. The derivation of $V(t)$ on $t$ is

$$
\begin{aligned}
\dot{V}(t)= & 2 \eta^{T}(t) P E_{1} \dot{\eta}(t)+\tau \dot{e}^{T}(t) Q \dot{e}(t) \\
& -\int_{t-\tau}^{t} \dot{e}^{T}(s) Q \dot{e}(s) d s+\theta \dot{e}^{T}(t) \Theta \dot{e}(t) \\
& -\int_{t-\theta}^{t} \dot{e}^{T}(s) \Theta \dot{e}(s) d s \\
= & 2 \eta^{T}(t) P B \eta(t)+2 \eta^{T}(t) P\left(\begin{array}{c}
p(t) \\
0
\end{array}\right) \\
& +2 \eta^{T}(t) P R \int_{t-\tau}^{t} \dot{\eta}(s) d s+\tau \dot{e}^{T}(t) Q \dot{e}(t) \\
& -\int_{t-\tau}^{t} \dot{e}^{T}(s) Q \dot{e}(s) d s+\theta \dot{e}^{T}(t) \Theta \dot{e}(t) \\
& -\int_{t-\theta}^{t} \dot{e}^{T}(s) \Theta \dot{e}(s) d s .
\end{aligned}
$$

From Lemma 2, we have

$$
\begin{aligned}
\dot{V}(t) \leq & \eta^{T}(t)\left(P B+B^{T} P^{T}\right) \eta(t)+2 \eta^{T}(t) P\left(\begin{array}{c}
p(t) \\
0
\end{array}\right) \\
& +2 \eta^{T}(t) P\left(\begin{array}{c}
q(t) \\
0
\end{array}\right)+2 \eta^{T}(t) P R \int_{t-\tau}^{t} \dot{\eta}(s) d s \\
& +\tau \dot{e}^{T}(t) Q \dot{e}(t) \\
& -\frac{1}{\tau}\left(\int_{t-\tau}^{t} \dot{e}^{T}(s) d s\right)^{T} Q\left(\int_{t-\tau}^{t} \dot{e}^{T}(s) d s\right) \\
& +\theta \dot{e}^{T}(t) \Theta \dot{e}(t) \\
& -\frac{1}{\theta}\left(\int_{t-\theta}^{t} \dot{e}^{T}(s) d s\right)^{T} \Theta\left(\int_{t-\theta}^{t} \dot{e}(s) d s\right) \\
= & \varsigma^{T}(t) \Pi \varsigma(t),
\end{aligned}
$$

where

$$
\Pi=\left(\begin{array}{cccccc}
P B+B^{T} P^{T} & 0 & P & P & P R & 0 \\
* & \tau Q & 0 & 0 & 0 & 0 \\
* & * & 0 & 0 & 0 & 0 \\
* & * & * & 0 & 0 & 0 \\
* & * & * & * & -\frac{1}{\tau} Q & 0 \\
* & * & * & * & * & -\frac{1}{\theta} \Theta
\end{array}\right) .
$$

From model (3), we have

$$
\begin{aligned}
0= & 2\left(Q_{1} e(t)+Q_{2} \dot{e}(t)+Q_{3} \int_{t-\tau}^{t} \dot{\eta}(s) d s\right)^{T}(-\dot{e}(t) \\
& +(A-L C, E-L D) \eta(t)+p(t)+q(t) \\
& \left.+(L C, L D) \int_{t-\tau}^{t} \dot{\eta}(s) d s\right)=\varsigma^{T}(t) \Xi \varsigma(t)
\end{aligned}
$$

where

$$
\begin{aligned}
& \Xi=\left(\begin{array}{cccccc}
\Xi_{11} & \Xi_{12} & \Xi_{13} & \Xi_{13} & \Xi_{14} & 0 \\
* & \Xi_{22} & \Xi_{23} & \Xi_{23} & \Xi_{24} & 0 \\
* & * & \Xi_{33} & 0 & \Xi_{34} & 0 \\
* & * & * & \Xi_{33} & \Xi_{34} & 0 \\
* & * & * & * & \Xi_{44} & 0 \\
* & * & * & * & * & 0
\end{array}\right), \\
& \Xi_{11}=\left(\begin{array}{c}
Q_{1}^{T} \\
0
\end{array}\right)(A-L C, E-L D)+\left(\left(\begin{array}{c}
Q_{1}^{T} \\
0
\end{array}\right)(A-L C, E-L D)\right)^{T} \text {, } \\
& \Xi_{12}=-\left(\begin{array}{c}
Q_{1}^{T} \\
0
\end{array}\right)+\left(Q_{2}^{T}(A-L C, E-L D)\right)^{T}, \\
& \Xi_{13}=\left(\begin{array}{c}
Q_{1}^{T} \\
0
\end{array}\right)\left(I, R_{8}\right) \\
& \Xi_{14}=\left(\begin{array}{c}
Q_{1}^{T} \\
0
\end{array}\right)(L C, L D)+\left(Q_{3}^{T}(A-L C, E-L D)\right)^{T}, \\
& \Xi_{22}=-\left(Q_{2}+Q_{2}^{T}\right), \\
& \Xi_{23}=Q_{2}^{T}\left(I, R_{9}\right), \\
& \Xi_{24}=-Q_{3}+Q_{2}^{T}(L C, L D)^{T}, \\
& \Xi_{33}=0 \text {, } \\
& \Xi_{34}=Q_{3}^{T}\left(I, R_{9}\right), \\
& \Xi_{44}=Q_{3}^{T}(L C, L D)+\left(Q_{3}^{T}(L C, L D)\right)^{T},
\end{aligned}
$$

where the proper dimension matrices $R_{9} \in R^{n \times n}$ is arbitrary. From formulas (42), (44), (48), and (50), we get

$$
\dot{V}(t) \leq \varsigma^{T}(t)(\Lambda+\Pi+\Xi) \varsigma(t)=\varsigma^{T}(t) \Omega \varsigma(t),
$$


where

$$
\begin{aligned}
& \Omega=\Lambda+\Pi+\Xi=\left(\begin{array}{cc}
\Omega_{11} & \Omega_{12} \\
* & \Omega_{22}
\end{array}\right) \\
& P B+B^{T} P^{T}=\left(\begin{array}{cc}
P_{1}(A-L C) & P_{1}(E-L D)-\delta P_{2} \\
0 & -\delta P_{3}
\end{array}\right)+\left(\begin{array}{cc}
P_{1}(A-L C) & P_{1}(E-L D)-\delta P_{2} \\
0 & -\delta P_{3}
\end{array}\right)^{T} \\
& +\delta\left(\begin{array}{cc}
P_{2} H \int_{0}^{1} F((1-\lambda) x(t)+\lambda \widehat{x}(t)) d \lambda N & 0 \\
P_{3} H \int_{0}^{1} F((1-\lambda) x(t)+\lambda \widehat{x}(t)) d \lambda N & 0
\end{array}\right)+\delta\left(\begin{array}{ccc}
P_{2} H \int_{0}^{1} F((1-\lambda) x(t)+\lambda \widehat{x}(t)) d \lambda N & 0 \\
P_{3} H \int_{0}^{1} F((1-\lambda) x(t)+\lambda \widehat{x}(t)) d \lambda N & 0
\end{array}\right)^{T} \\
& =Z+Z^{T}+\delta\left(\begin{array}{c}
P_{2} H \\
P_{3} H
\end{array}\right) \int_{0}^{1} F((1-\lambda) x(t)+\lambda \widehat{x}(t)) d \lambda\left(\begin{array}{ll}
N & 0
\end{array}\right) \\
& +\delta\left[\left(\begin{array}{l}
P_{2} H \\
P_{3} H
\end{array}\right) \int_{0}^{1} F((1-\lambda) x(t)+\lambda \hat{x}(t)) d \lambda\left(\begin{array}{ll}
N & 0
\end{array}\right)\right]^{T},
\end{aligned}
$$

where $Z=\left(\begin{array}{cc}P_{1}(A-L C) & P_{1}(E-L D)-\delta P_{2} \\ 0 & -\delta P_{3}\end{array}\right)$.

From Lemma 3, we obtain that $S_{1}+S_{1}^{T}+\Xi_{11}+P B+B^{T} P^{T}<$ 0 is equivalent to

$$
\begin{aligned}
S_{1}+S_{1}^{T}+\Xi_{11}+Z+Z^{T} & \\
& +\varepsilon^{-1} \delta^{2}\left(\begin{array}{cc}
P_{2} H H^{T} P_{2}^{T} & P_{2} H H^{T} P_{3}^{T} \\
* & P_{3} H H^{T} P_{3}^{T}
\end{array}\right) \\
& +\varepsilon\left(\begin{array}{cc}
N^{T} N & 0 \\
0 & 0
\end{array}\right)<0 .
\end{aligned}
$$

Based on the above derivation, we have the following result.

Theorem 9. The fault tolerant synchronization (3) and (6) is achieved if there exist constants $\tau, \varepsilon>0, \delta>0$, the positive definite matrices $P_{1}=P_{1}^{T}>0, Q=Q^{T}>0$, and the matrices $Q_{j}, M_{j}, U_{j}, j=1,2, Q_{j}, M_{j}, U_{j}, j=1,2$, $L, C, D, R_{i}, i=1,2, \ldots, 9$, such that matrix $\Sigma<0$, where

$$
\begin{aligned}
\Sigma= & \left(\begin{array}{cc}
\Sigma_{11} & \Omega_{12} \\
* & \Omega_{22}
\end{array}\right), \\
\Theta_{11}= & S_{1}+S_{1}^{T}+\Xi_{11}+Z+Z^{T} \\
& +\varepsilon^{-1} \delta^{2}\left(\begin{array}{cc}
P_{2} H H^{T} P_{2}^{T} & P_{2} H H^{T} P_{3}^{T} \\
* & P_{3} H H^{T} P_{3}^{T}
\end{array}\right) \\
& +\varepsilon\left(\begin{array}{cc}
N^{T} N & 0 \\
0 & 0
\end{array}\right) .
\end{aligned}
$$

Remark 10. After extending system (37) to singular system (40) by the transformation $e(t) \rightarrow \eta(t)$, taking proper matrices $R_{i}, i=1,2, \ldots, 9$, and the constant $\varepsilon>0$ can 
decrease the conservation of stability of error system (37) by constructing the Lyapunov functional

$$
\begin{aligned}
V(t)= & e^{T}(t) P_{1} e(t)+\int_{-\tau}^{0} \int_{t+r}^{t} \dot{e}^{T}(s) Q \dot{e}(s) d s d r \\
& +\int_{-\theta}^{0} \int_{t+r}^{t} \dot{e}^{T}(s) \Theta \dot{e}(s) d s d r \\
= & \eta^{T}(t) P E_{1} \eta(t)+\int_{-\tau}^{0} \int_{t+\theta}^{t} \dot{e}^{T}(s) Q \dot{e}(s) d s d \theta \\
& +\int_{-\theta}^{0} \int_{t+r}^{t} \dot{e}^{T}(s) \Theta \dot{e}(s) d s d r .
\end{aligned}
$$

When $h(x(t-\theta))=0$, inequality (26) is transformed to

$$
\omega^{T}(t) \Lambda \omega(t) \geq 0,
$$

where

$\omega(t)$

$$
\begin{aligned}
& =\left(\begin{array}{ll}
\eta^{T}(t) & \dot{e}^{T}(t)
\end{array}\left(\begin{array}{c}
p(t) \\
0
\end{array}\right)^{T}\left(\int_{t-\tau}^{t} \dot{e}(s) d s\right)^{T}\right)^{T}, \\
& \Lambda=\left(\begin{array}{cccc}
S_{1}+S_{1}^{T} & 0 & S_{2}+S_{3}^{T} & 0 \\
* & 0 & 0 & 0 \\
* & * & S_{4}+S_{4}^{T} & 0 \\
* & * & * & 0
\end{array}\right) \\
& S_{1}=\left(\begin{array}{cc}
-\varepsilon U_{2}^{T} M_{2} & 0 \\
0 & 0
\end{array}\right), \\
& S_{2}=\left(\begin{array}{cc}
\varepsilon U_{2}^{T} M_{1} & \varepsilon R_{1} \\
0 & \varepsilon R_{2}
\end{array}\right) \text {, } \\
& S_{3}=\left(\begin{array}{cc}
\varepsilon U_{1}^{T} M_{2} & 0 \\
\varepsilon R_{3} & \varepsilon R_{4}
\end{array}\right) \text {, } \\
& S_{4}=\left(\begin{array}{cc}
-\varepsilon U_{1}^{T} M_{1} & \varepsilon R_{5} \\
\varepsilon R_{6} & \varepsilon R_{7}
\end{array}\right) \text {, } \\
& \varepsilon>0
\end{aligned}
$$

is any constant. The proper dimension matrices $R_{i} \in R^{n \times n}$, $i=1,2, \ldots, 7$, are arbitrary.

We choose Lyapunov function

$$
\begin{aligned}
V(t) & =e^{T}(t) P_{1} e(t)+\int_{-\tau}^{0} \int_{t+\theta}^{t} \dot{e}^{T}(s) Q \dot{e}(s) d s d \theta \\
& =\eta^{T}(t) P E_{1} \eta(t)+\int_{-\tau}^{0} \int_{t+\theta}^{t} \dot{e}^{T}(s) Q \dot{e}(s) d s d \theta,
\end{aligned}
$$

where $P=\left(\begin{array}{cc}P_{1} & P_{2} \\ 0 & P_{3}\end{array}\right)$, and it is easy to know that $P E_{1}=E_{1}^{T} P^{T}$, and differentiating $V(t)$ with respect to $t$ and using Lemma 2 yield

$$
\dot{V}(t) \leq \omega^{T}(t) \Pi \omega(t),
$$

where

$$
\Pi=\left(\begin{array}{cccc}
P B+B^{T} P^{T} & 0 & P & P R \\
* & \tau Q & 0 & 0 \\
* & * & 0 & 0 \\
* & * & * & -\frac{1}{\tau} Q
\end{array}\right) .
$$

From model (12), we have

$$
\begin{aligned}
0 & =2\left(Q_{1} e(t)+Q_{2} \dot{e}(t)+Q_{3} \int_{t-\tau}^{t} \dot{\eta}(s) d s\right)^{T}(-\dot{e}(t) \\
& +(A-L C, E-L D) \eta(t)+p(t) \\
& \left.+(L C, L D) \int_{t-\tau}^{t} \dot{\eta}(s) d s\right)=\sigma^{T}(t) \Xi \sigma(t),
\end{aligned}
$$

where

$$
\begin{aligned}
& \Xi=\left(\begin{array}{cccc}
\Xi_{11} & \Xi_{12} & \Xi_{13} & \Xi_{14} \\
* & \Xi_{22} & \Xi_{23} & \Xi_{24} \\
* & * & \Xi_{33} & \Xi_{34} \\
* & * & * & \Xi_{44}
\end{array}\right), \\
& \Xi_{11}=\left(\begin{array}{c}
Q_{1}^{T} \\
0
\end{array}\right)(A-L C, E-L D)+\left(\left(\begin{array}{c}
Q_{1}^{T} \\
0
\end{array}\right)(A-L C, E-L D)\right)^{T} \text {, } \\
& \Xi_{12}=-\left(\begin{array}{c}
Q_{1}^{T} \\
0
\end{array}\right)+\left(Q_{2}^{T}(A-L C, E-L D)\right)^{T}, \\
& \Xi_{13}=\left(\begin{array}{c}
Q_{1}^{T} \\
0
\end{array}\right)\left(I, R_{8}\right) \\
& \Xi_{14}=\left(\begin{array}{c}
Q_{1}^{T} \\
0
\end{array}\right)(L C, L D)+\left(Q_{3}^{T}(A-L C, E-L D)\right)^{T}, \\
& \Xi_{22}=-\left(Q_{2}+Q_{2}^{T}\right), \\
& \Xi_{23}=Q_{2}^{T}\left(I, R_{8}\right) \\
& \Xi_{24}=-Q_{3}+Q_{2}^{T}(L C, L D)^{T}, \\
& \Xi_{33}=0 \text {, } \\
& \Xi_{34}=Q_{3}^{T}\left(I, R_{8}\right) \text {, } \\
& \Xi_{44}=Q_{3}^{T}(L C, L D)+\left(Q_{3}^{T}(L C, L D)\right)^{T},
\end{aligned}
$$

where the proper dimension matrices $R_{8} \in R^{n \times n}$ is arbitrary.

Based on the above derivation, we have the following result.

Corollary 11. The fault tolerant synchronization (3) and (5) is achieved if there exist constants $\tau, \varepsilon>0, \delta>0$, the positive definite matrices $P_{1}=P_{1}^{T}>0, Q=Q^{T}>0$, and 

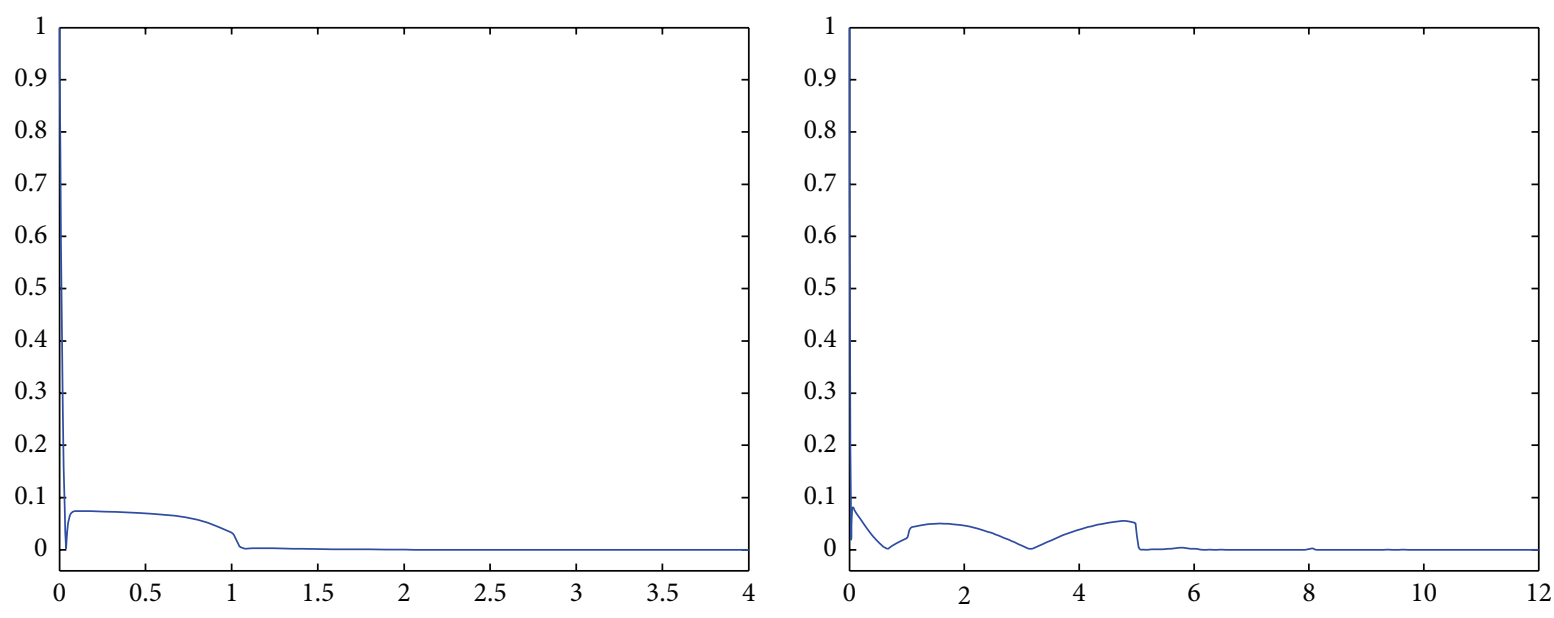

FIGURE 1: The state responses of $x(t)$ and $\widehat{x}(t)$ when $f(t)$ and $\widehat{f}(t)$ are derivable on $t$.

the matrices $Q_{j}, M_{j}, U_{j}, j=1,2, Q_{j}, M_{j}, U_{j}, j=1,2$, $L, C, D, R_{i}, i=1,2, \ldots, 8$, such that matrix $\Theta<0$, where

$$
\begin{aligned}
& \Theta=\left(\begin{array}{cc}
\Theta_{11} & \Omega_{12} \\
* & \Omega_{22}
\end{array}\right), \\
& \Theta_{11}=S_{1}+S_{1}^{T}+\Xi_{11}+Z+Z^{T} \\
& +\varepsilon^{-1} \delta^{2}\left(\begin{array}{cc}
P_{2} H H^{T} P_{2}^{T} & P_{2} H H^{T} P_{3}^{T} \\
* & P_{3} H H^{T} P_{3}^{T}
\end{array}\right) \\
& +\varepsilon\left(\begin{array}{cc}
N^{T} N & 0 \\
0 & 0
\end{array}\right) \text {. }
\end{aligned}
$$

\section{Numerical Examples}

Example 1. Consider a typical delayed Hopfield neural networks [29-32] with two neurons

$$
\begin{aligned}
A & =-I, \\
g(x(t)) & =\left(\begin{array}{cc}
2.0 & -0.1 \\
-5.0 & 3.0
\end{array}\right)\left(\begin{array}{l}
\tanh \left(x_{1}(t)\right) \\
\tanh \left(x_{2}(t)\right)
\end{array}\right), \\
h(x(t-\theta)) & =\left(\begin{array}{cc}
0.15 & -0.1 \\
-0.2 & -2.5
\end{array}\right)\left(\begin{array}{l}
\tanh \left(x_{1}(t-\theta)\right) \\
\tanh \left(x_{2}(t-\theta)\right)
\end{array}\right), \\
\tau & =0.01, \\
\theta & =1, \\
L C & =L D=-38 I, \\
E & =2 I, \\
x(t) & =\left(\begin{array}{l}
x_{1} \\
x_{2}
\end{array}\right) .
\end{aligned}
$$

When $f(t)$ and $\widehat{f}(t)$ are derivable on $t$, we take

$$
\begin{aligned}
& f(t)= \begin{cases}(0,0)^{T}, & 0 \leq t \leq 2 \\
(0,2 \sin t)^{T}, & t \geq 2,\end{cases} \\
& \dot{\tilde{f}}(t)=\left(\begin{array}{ll}
0.2 & 0 \\
0 & 0
\end{array}\right)\left(\begin{array}{l}
x_{1}(t)-x_{3}(t) \\
x_{2}(t)-x_{4}(t)
\end{array}\right)-2 \widetilde{f}(t) .
\end{aligned}
$$

Simulation results are shown in Figure 1.

When $f(t)$ and $\widehat{f}(t)$ are derivable on $x(t)$, we take

$$
\begin{aligned}
& f(t) \\
& \quad= \begin{cases}(0,0)^{T}, & 0 \leq t \leq 10 \\
\left(0.2 \sin \left(x_{1}(t)\right), 0.4 \sin \left(x_{2}(t)\right)\right)^{T}, & t \geq 10 .\end{cases}
\end{aligned}
$$

Simulation results are shown in Figure 2.

Example 2. Consider chaotic Lü system [31, 32]; that is,

$$
\begin{aligned}
A & =\left(\begin{array}{ccc}
-36 & 36 & 0 \\
0 & 20 & 0 \\
0 & 0 & -3
\end{array}\right), \\
g(x(t)) & =\left(\begin{array}{c}
0 \\
-x_{1} x_{3} \\
x_{1} x_{2}
\end{array}\right),
\end{aligned}
$$

where $x(t)=\left(\begin{array}{l}x_{1} \\ x_{2} \\ x_{3}\end{array}\right)$. We choose

$$
\begin{aligned}
& L C=\left(\begin{array}{ccc}
0 & -38 & 0 \\
0 & -38 & 0 \\
0 & 0 & 0
\end{array}\right), \\
& L D=\left(\begin{array}{ccc}
0 & 0 & 0 \\
0 & -38 & 0 \\
0 & 0 & 0
\end{array}\right),
\end{aligned}
$$



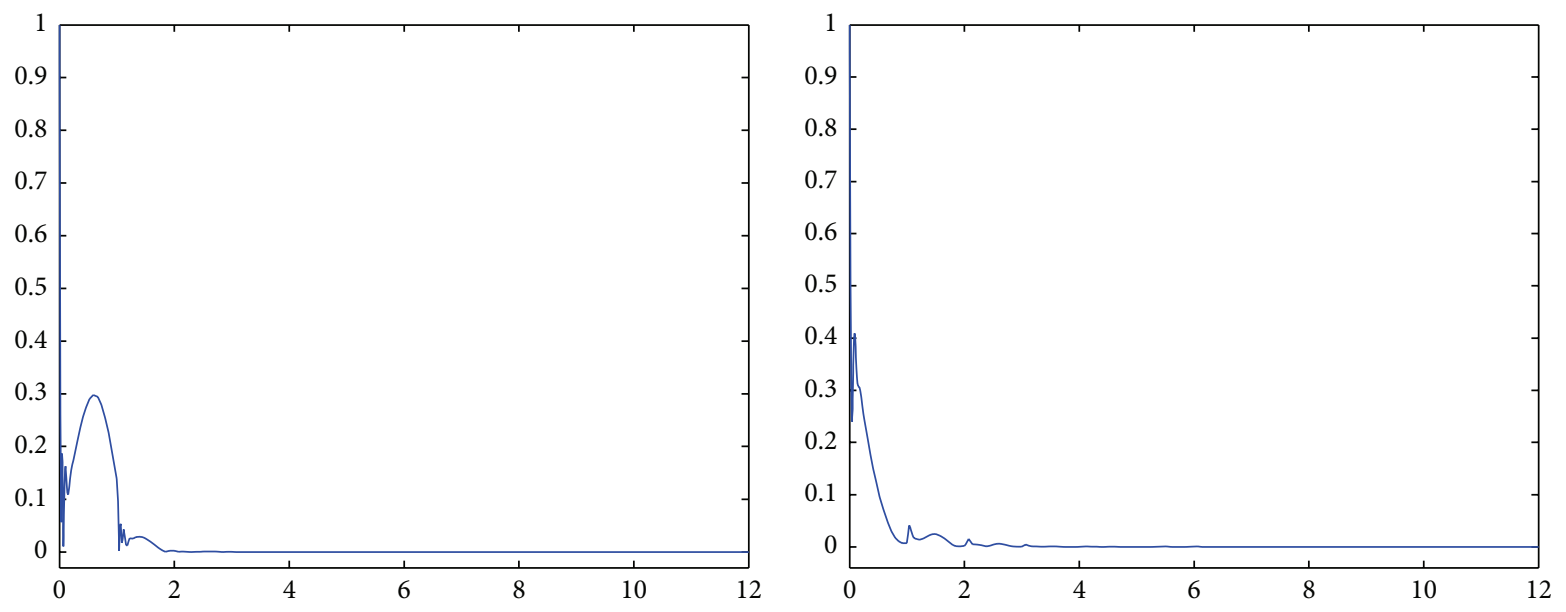

FIGURE 2: The state responses of $x(t)$ and $\widehat{x}(t)$ when $f(t)$ and $\widehat{f}(t)$ are derivable on $x(t)$.
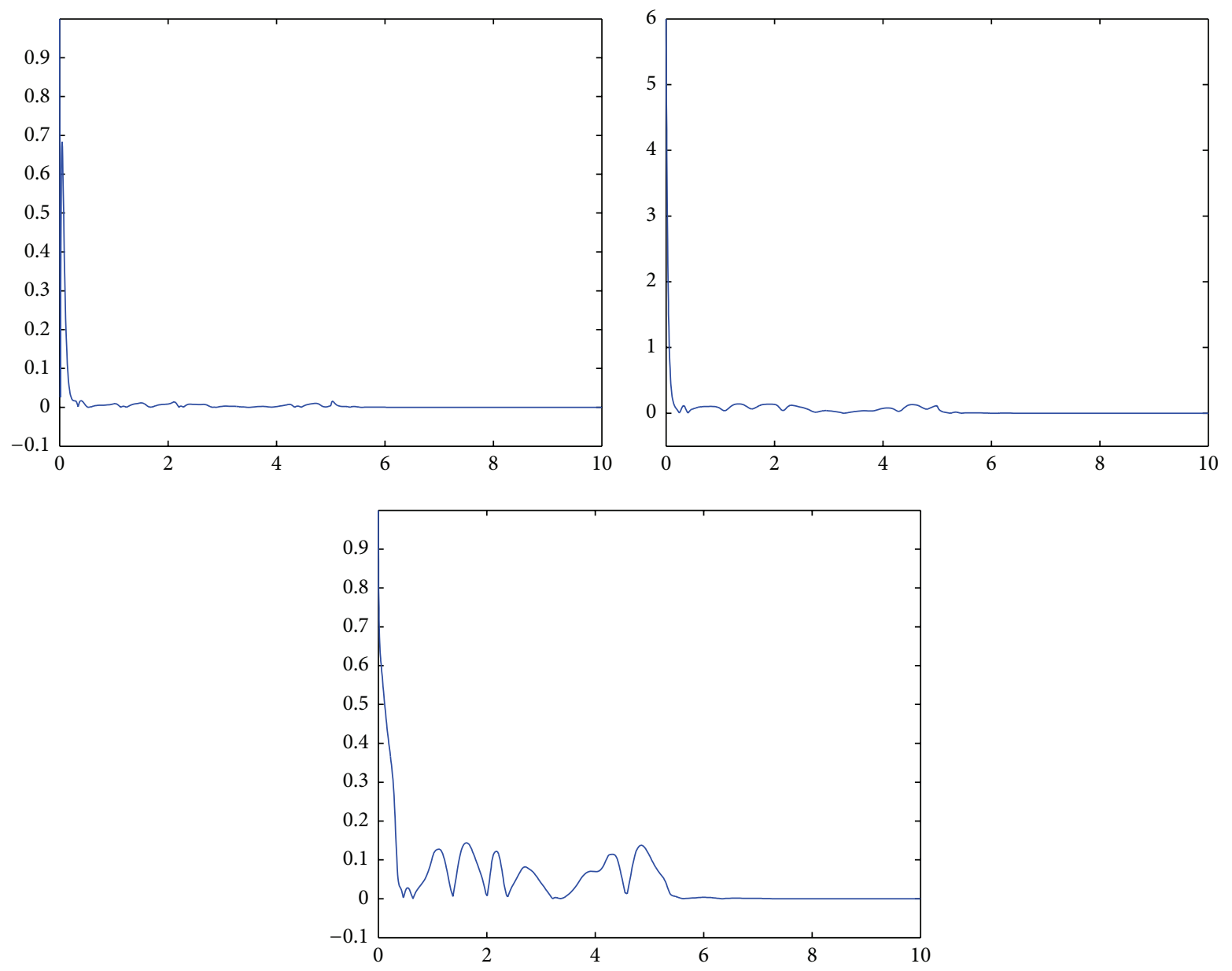

Figure 3: The state responses of $x(t)$ and $\widehat{x}(t)$ when $f(t)$ and $\widehat{f}(t)$ are derivable on $t$.

$$
\begin{aligned}
& E=\left(\begin{array}{lll}
2 & 0 & 0 \\
0 & 2 & 0 \\
0 & 0 & 2
\end{array}\right), \\
& \tau=0.01 .
\end{aligned}
$$

When $f(t)$ and $\widehat{f}(t)$ are derivable on $t$, we take

$$
f(t)= \begin{cases}(0,0,0)^{T}, & 0 \leq t \leq 2 \\ (0,2 \sin t, 0)^{T}, & t \geq 2\end{cases}
$$



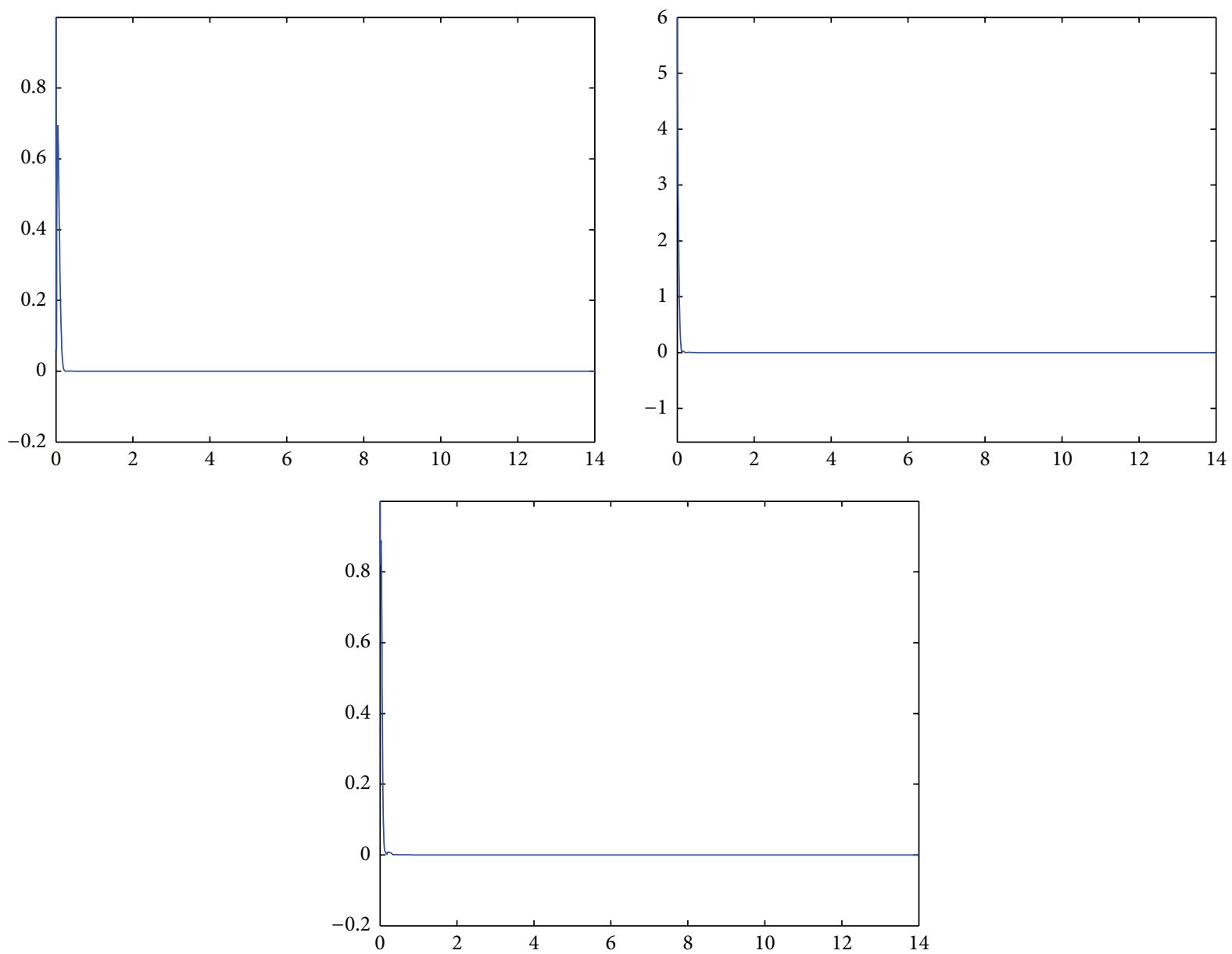

FIGURE 4: The state responses of $x(t)$ and $\widehat{x}(t)$ when $f(t)$ and $\widehat{f}(t)$ are derivable on $x(t)$.

$$
\dot{\tilde{f}}(t)=\left(\begin{array}{cc}
0.2 & 0 \\
0 & 0
\end{array}\right)\left(\begin{array}{l}
x_{1}(t)-x_{3}(t) \\
x_{2}(t)-x_{4}(t)
\end{array}\right)-2 \tilde{f}(t) .
$$

Simulation results are shown in Figure 3.

When $f(t)$ and $\widehat{f}(t)$ are derivable on $x(t)$, we take

$$
\begin{aligned}
& f(t) \\
& = \begin{cases}(0,0,0)^{T}, & 0 \leq t \leq 5 \\
\left(0,0.4 \sin \left(x_{1}(t)\right), 0.4 \sin \left(x_{2}(t)\right)\right)^{T}, & t \geq 5 .\end{cases}
\end{aligned}
$$

Simulation results are shown in Figure 4.

\section{Conclusions}

In this paper, taking into account the constant propagation delay and actuator faults and the extended transformation of the error systems, the problem of the chaotic fault tolerant synchronization has been addressed. The conservation of stability of the error systems is lower than that of other existing literatures.

\section{Conflict of Interests}

The author declares that there is no conflict of interests regarding the publication of this paper.

\section{Acknowledgments}

This work is supported by the National Natural Science Foundation of China (11071141), the Natural Science Foundation of Shandong Province of China (ZR2011AL018, ZR2011AQ008), a Project of Shandong Province Higher Educational Science and Technology Program (J13LI02, J11LA06), and the Research Fund Project of Heze University (XY10KZ01).

\section{References}

[1] M. A. Aziz-Alaoui, "A survey of chaotic synchronization and secure communication," in Proceedings of the 12th IEEE International Conference on Electronics, Circuits and Systems (ICECS '05), pp. 1-4, IEEE, December 2005.

[2] L. M. Pecora and T. L. Carroll, "Synchronization in chaotic systems," Physical Review Letters, vol. 64, no. 8, pp. 821-824, 1990.

[3] Q. Zhang, J. Zhou, and G. Zhang, "Stability concerning partial variables for a class of time-varying systems and its application 
in chaos synchronization," in Proceeding of the 24th Chinese Control Conference, pp. 135-139, South China University of Technology Press, Guangzhou, China, 2005.

[4] X. Wang, X. Wu, Y. He, and G. Aniwar, "Chaos synchronization of Chen system and its application to secure communication," International Journal of Modern Physics B, vol. 22, no. 21, pp. 3709-3720, 2008.

[5] Q.-L.Zhang, "Synchronization of multi-chaotic systems via ring impulsive control," Control Theory \& Applications, vol. 27, no. 2, pp. 226-232, 2010.

[6] Q. Zhang, "Synchronization analysis of multiple systems based on a class of symmetric matrix," Bio Technology, vol. 10, no. 23, pp. 14496-14502, 2014.

[7] W.-H. Chen, Z. P. Wang, and X. M. Lu, "On sampled-data control for master-slave synchronization of chaotic Lur'e systems," IEEE Transactions on Circuits and Systems II: Express Briefs, vol. 59, no. 8, pp. 515-519, 2012.

[8] S. J. Theesar, S. Banerjee, and P. Balasubramaniam, "Synchronization of chaotic systems under sampled-data control," Nonlinear Dynamics. An International Journal of Nonlinear Dynamics and Chaos in Engineering Systems, vol. 70, no. 3, pp. 1977-1987, 2012.

[9] J. Joomwong and V. N. Phat, "Adaptive control with two controllers of the Lu's system," Journal of Interdisciplinary Mathematics, vol. 13, no. 2, pp. 211-215, 2010.

[10] K. Ratchagit and V. N. Phat, "Synchronization of Lu's system using active control," International Journal of Applied Mathematics \& Statistics, vol. 18, no. S10, pp. 36-40, 2010.

[11] K. Ratchagit, "A novel adaptive synchronization of Lu's chaotic system," European Journal of Scientific Research, vol. 45, no. 4, pp. 630-636, 2010.

[12] M. Rajchakit, P. Niamsup, and G. Rajchakit, "A switching rule for exponential stability of switched recurrent neural networks with interval time-varying delay," Advances in Difference Equations, vol. 2013, article 44, 2013.

[13] P. Niamsup, K. Ratchagit, and V. N. Phat, "Novel criteria for finite-time stabilization and guaranteed cost control of delayed neural networks," Neurocomputing, vol. 160, pp. 281-286, 2015.

[14] Y. P. Zhang and J. T. Sun, "Impulsive robust fault-tolerant feedback control for chaotic Lure systems," Chaos, Solitons \& Fractals, vol. 39, no. 3, pp. 1440-1446, 2009.

[15] F. Huang, J. Wang, and J. Wang, "Adaptive observer based fault-tolerant chaotic synchronization comunication design," in Proceedings of the Chinese Control and Decision Conference (CCDC '11), pp. 2894-2897, IEEE, Mianyang, China, May 2011.

[16] D.-Z. Ma, H.-G. Zhang, Z.-S. Wang, and J. Feng, "Fault tolerant synchronization of chaotic systems based on T-S fuzzy model with fuzzy sampled-data controller," Chinese Physics B, vol. 19, no. 5, Article ID 050506, 2010.

[17] Q. L. Hu and B. Xiao, "Fault-tolerant sliding mode attitude control for flexible spacecraft under loss of actuator effectiveness," Nonlinear Dynamics, vol. 64, no. 1-2, pp. 13-23, 2011.

[18] D. Chen, X. Huang, and T. Ren, "Study on chaotic fault tolerant synchronization control based on adaptive observer," The Scientific World Journal, vol. 2014, Article ID 405396, 5 pages, 2014.

[19] Q. Shen, B. Jiang, and V. Cocquempot, "Adaptive fault tolerant synchronization with unknown propagation delays and actuator faults," International Journal of Control, Automation and Systems, vol. 10, no. 5, pp. 883-889, 2012.
[20] D. Ye and X. Zhao, "Robust adaptive synchronization for a class of chaotic systems with actuator failures and nonlinear uncertainty," Nonlinear Dynamics, vol. 76, no. 2, pp. 973-983, 2014.

[21] J. E. Stellet and T. Rogg, "On linear observers and application to fault detection in synchronous generators," Control Theory and Technology, vol. 12, no. 4, pp. 345-356, 2014.

[22] C. P. Tan and C. Edwards, "Sliding mode observers for reconstruction of simultaneous actuator and sensor faults," in Proceedings of the 42nd IEEE Conference on Decision and Control, vol. 2, pp. 1455-1460, IEEE, New York, NY, USA, December 2003.

[23] H. Tao, D. Chen, and H. Yang, "Iterative learning fault diagnosis algorithm for nonlinear systems based on extend filter," Control and Decision, vol. 30, no. 6, pp. 1027-1032, 2015.

[24] M. Rajchakit, P. Niamsup, and G. Rajchakit, "A constructive way to design a switching rule and switching regions to mean square exponential stability of switched stochastic systems with non-differentiable and interval time-varying delay," Journal of Inequalities and Applications, vol. 2013, article 499, 2013.

[25] J. Sun and J. Chen, Stability Analysis and Application of TimeDelay Systems, Science Press, Beijing, China, 2012.

[26] I. R. Petersen and C. V. Hollot, "A Riccati equation approach to the stabilization of uncertain linear systems," Automatica, vol. 22, no. 4, pp. 397-411, 1986.

[27] C. E. de Souza and X. Li, "Delay-dependent robust $H_{\infty}$ control of uncertain linear state-delayed systems," Automatica, vol. 35, no. 7, pp. 1313-1329, 1999.

[28] J. Wang, Q. Zhang, and D. Xiao, "Output strictly passive control of uncertain singular neutral systems," Mathematical Problems in Engineering, vol. 2015, Article ID 591854, 12 pages, 2015.

[29] L. Xiang, J. Zhou, and Z. Liu, "On the asymptotic behavior of Hopfield neural networks with periodic inputs," Applied Mathematics and Mecjamocs, vol. 23, no. 12, pp. 1367-1373, 2002.

[30] K. Gopalsamy and X. Z. He, "Stability in asymmetric Hopfield nets with transmission delays," Physica D. Nonlinear Phenomena, vol. 76, no. 4, pp. 344-358, 1994.

[31] J. Lü and J. Lu, "Controlling uncertain Lü system using linear feedback," Chaos, Solitons and Fractals, vol. 17, no. 1, pp. 127-133, 2003.

[32] Y. Yu and S. Zhang, "Hopf bifurcation analysis of the Lü system," Chaos, Solitons and Fractals, vol. 21, no. 5, pp. 1215-1220, 2004. 


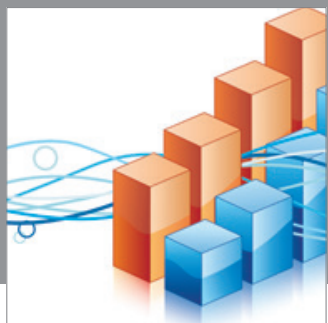

Advances in

Operations Research

mansans

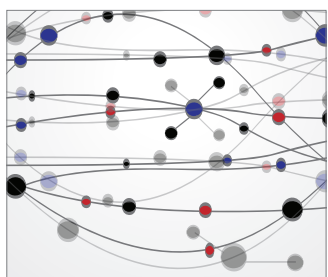

The Scientific World Journal
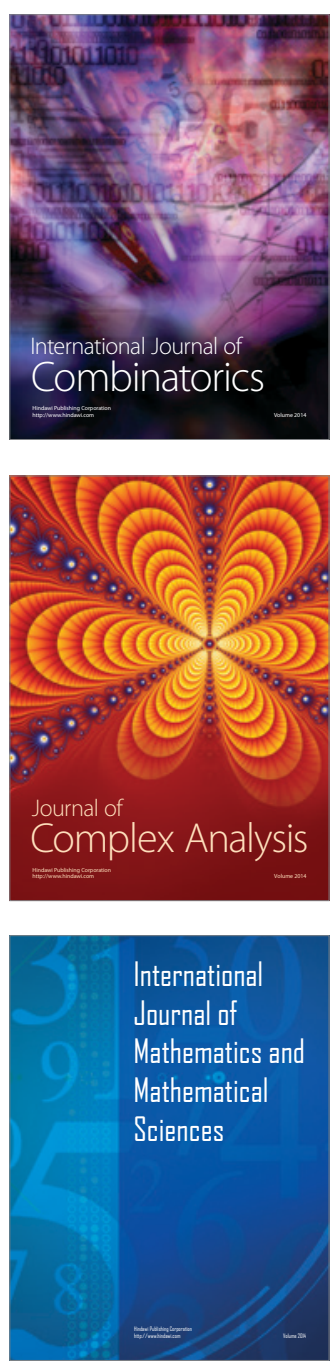
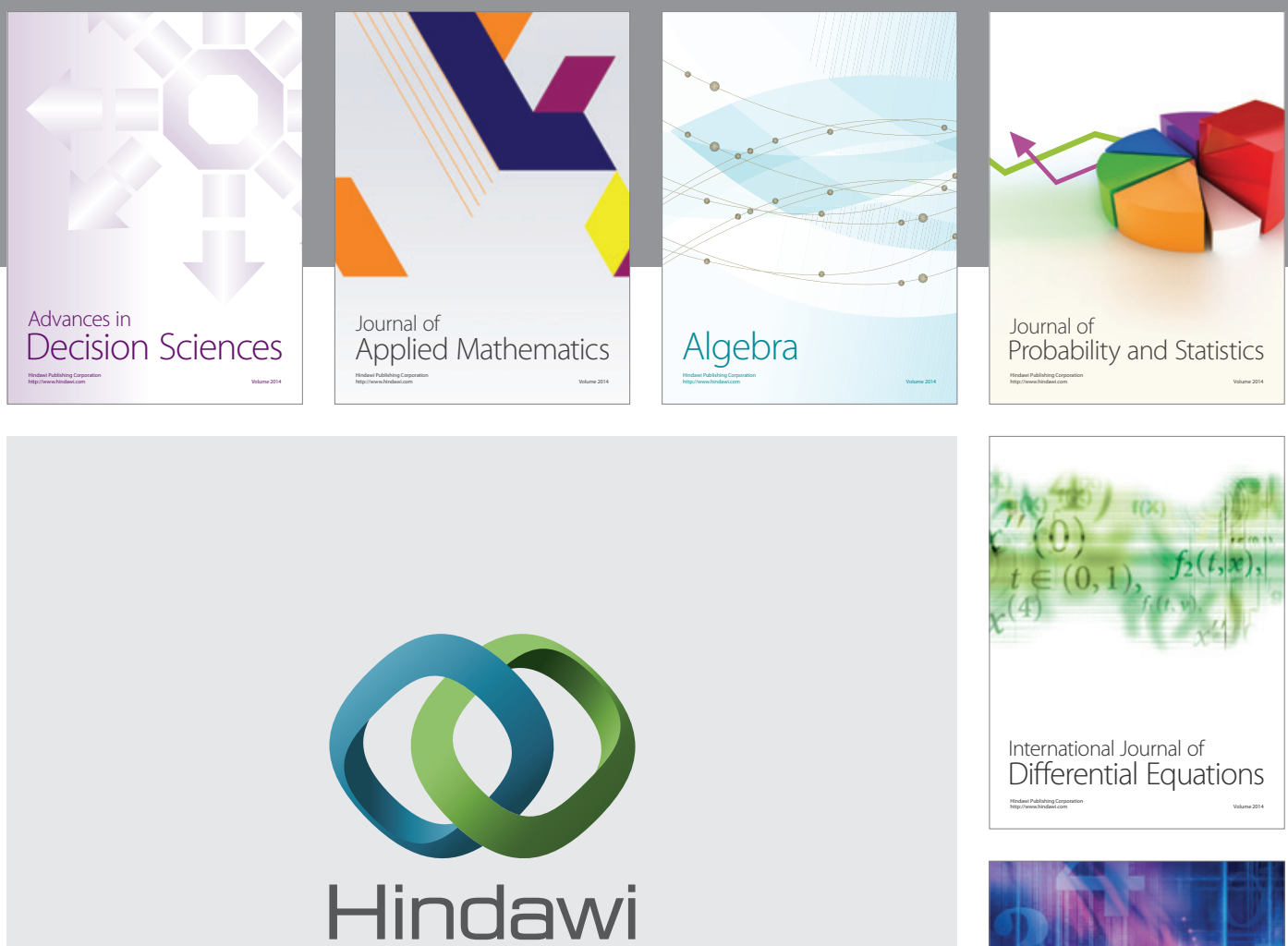

Submit your manuscripts at http://www.hindawi.com
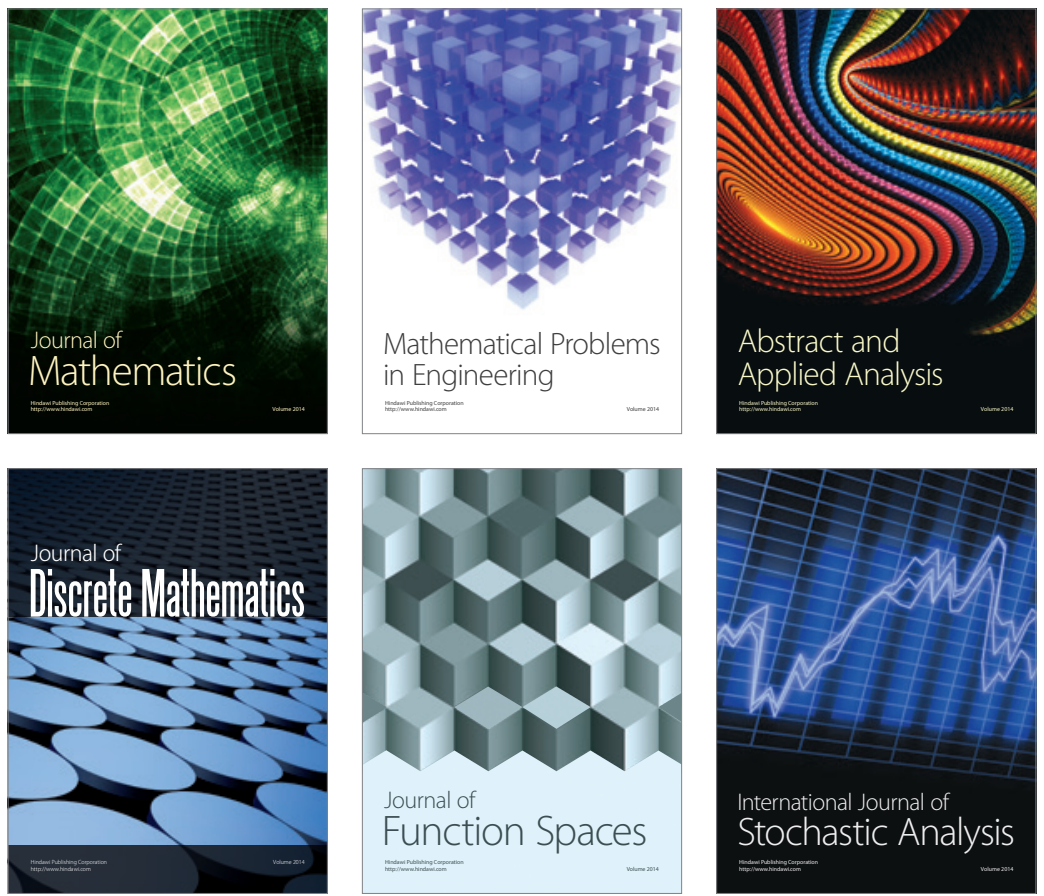

Journal of

Function Spaces

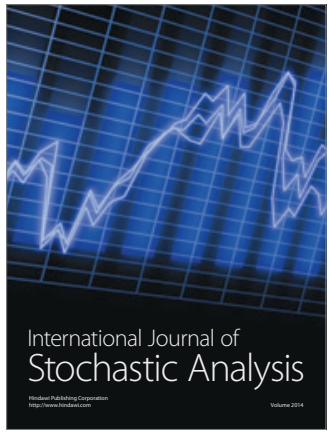

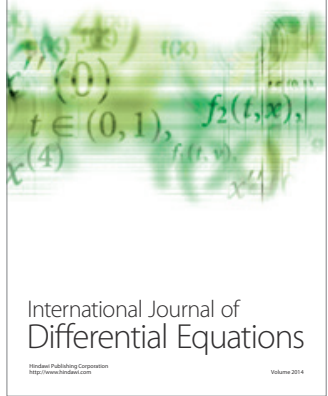
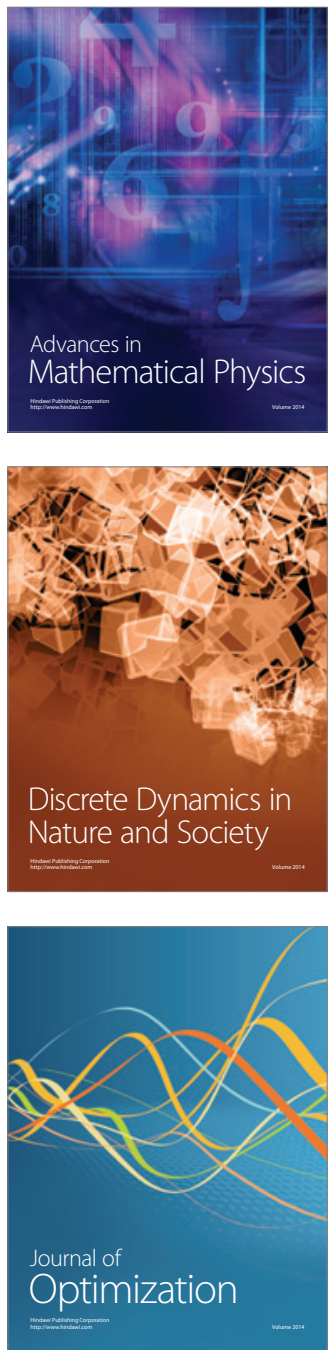